\section{Repercusiones en la cavidad oral por la utilización de piercing. Reporte de un caso}

\author{
Repercussions on oral cavity due to \\ piercing use. Case report
}

\begin{abstract}
Resumen
El piercing corporal se define como la penetración de un adorno a los orificios hechos en la piel y/o mucosa a través de un instrumento agudo que crea una abertura en torno a la cual es posible aplicar el ornamento. La utilización de piercing oral en jóvenes cada vez adquiere más fuerza debido a que se considera una expresión de arte en la cavidad oral. Dentro de las zonas más comunes para colocarlos están el dorso de la lengua y el labio inferior. Esta moda puede llegar a ser muy perjudicial para la salud oral ya que puede provocar infecciones y en el peor de los casos ocasionar complicaciones que coloquen en riesgo la vida. En el presente artículo se describe las características del dańo ocasionado en la cavidad bucal por la utilización de un piercing lingual. Se reporta un paciente masculino de 29 años con fractura coronal y dolor al frío y calor en el diente 3.6; además de pérdida ósea vertical en los dientes 4.1 y 4.6; el caso se resolvió a través de tratamiento endodóntico no quirúrgico y tratamiento periodontal. Se concluye que el uso de piercing lingual puede asociarse a fracturas coronales y problemas periodontales.
\end{abstract}

Palabras clave: Bolsa periodontal; Caries dental; Endodoncia; Lengua; Perforación del cuerpo.

\begin{abstract}
The corporal piercing is defined as the penetration of an ornament to holes made in the skin and/or mucosa, using an acute instrument that creates an opening through which the ornament can be applied. The use of oral piercing in young people is gaining strength because it is considered an expression of art in the oral cavity. Within the most common areas to place them are the back of the tongue and the lower lip. This fashion can become very harmful to oral health because it can cause infections and in the worst cases cause complications that place life at risk. In this article, we describe the characteristics of damage caused in the oral cavity for use of a tongue piercing. A 29-year-old male, patient with coronal fracture and pain to cold and heat in tooth 3.6 was reported, as well as vertical bone loss in teeth 4.1 and 4.6; the case was resolved through non-surgical endodontic treatment and referred to periodontics service. We conclude that the use of lingual piercing can be associated with coronal fractures and periodontal problems.
\end{abstract}

Keywords: Body piercing; Dental caries; Endodontics; Periodontal pocket; Tongue.
ISSN: 1560-9111

\section{Caso Clínico}

Alexander Villalobos Montero ${ }^{1, a}$, Mayid Barzuna Ulloa $^{1, b}$, Mariela Barzuna Pacheco ${ }^{1, b}$

\author{
${ }^{1}$ Universidad Latina. San José, Costa Rica. \\ a Cirujano Dentista.

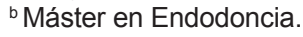

\section{Correspondencia:} \\ Alexander Villalobos Montero \\ Correo electrónico: alex.villalobos730@gmail. \\ com \\ Centro Médico Jehová Rafa. Desamparados. \\ San José, Costa Rica. \\ Coautores: \\ Mayid Barzuna Ulloa \\ endobarzuna@hotmail.com \\ Mariela Barzuna Pacheco \\ marielabarzuna@hotmail.com
}

Conflicto de intereses: Los autores declaran no tener conflictos de interés.

Fuente de financiamiento: Autofinanciado

Fecha de recepción: 16/11/17

Fecha de aceptación: 10/01/18 


\section{Introducción}

El piercing corporal se define como la penetración de un adorno a los orificios hechos en la piel y/o mucosa a través de un instrumento agudo que crea una abertura en torno a la cual es posible aplicar el ornamento ${ }^{1}$.

El uso de perforaciones en diferentes partes del cuerpo para llevar algún tipo de pendiente ha sido algo significativo en la historia ${ }^{2,3}$. En Egipto se colocaba en el ombligo como signo de nobleza; en la antigua Roma los centuriones los usaban en los pezones, en la India se colocan en la nariz antes del matrimonio mientras que los esquimales y Aleuts los utilizan en el labio inferior de las niñas como acto de purificación. Otras tribus como los Siux, Mayas y Aztecas también realizaban perforaciones. Los aztecas utilizaban expansores o pendientes, en especial los reyes. Existen algunas tribus en África y América que siguen usándolos por motivos religiosos o tribales ${ }^{4}$.

La realización de tatuajes, piercings, marcas con hierro y demás actualmente se conoce como "body art". El "body piercing” se ha utilizado hace unos 60 mil ańos ${ }^{1}$. Los materiales utilizados antiguamente incluían piedras, huesos o marfil. Actualmente se utilizan diferentes materiales como acero quirúrgico inoxidable, cobalto, aleaciones de cromo, plata, aleaciones de oro, titanio, además de materiales resinosos como el polimetilmetacrilato o el politetrafluoroetileno ${ }^{2}$.

Los piercings orales se han convertido en la forma más prevalente de arte corporal presentando actualmente una frecuencia entre el 3,4\% y el 20,3\% de uso ${ }^{5,6}$. Usualmente es practicado por personas inescrupulosas que desconocen el daño que podría estarse provocando, donde los profesionales en el área de la salud están lejos de esa práctica.

La Asociación Dental Americana (ADA) emitió una regulación para el uso de los piercings orales e inclusive en ciertos países se redactaron leyes para condicionar su uso obligando a seguir ciertas normas de control como botiquín de primeros auxilios, autorización de los municipios, vacunación contra la hepatitis $\mathrm{B}$ y tétanos y la utilización de material desechable por parte del personal que los aplica ${ }^{7,8}$.

Los piercings intraorales pueden colocarse en la lengua a nivel de la zona dorsoventral o dorsolateral ${ }^{9}$, el labio inferior es otro lugar muy frecuente de utilización; además, se pueden colocar en labios, mejillas, frenillo lingual y úvula ${ }^{1}$.

En 1994 se describió por primera vez las complicaciones asociadas al uso del piercing lingual ${ }^{10}$ presentándose las siguientes manifestaciones: lesiones vasculares ${ }^{11}$, inflamación ${ }^{1,11}$, dolor, lesiones nerviosas ${ }^{1}$, infecciones ${ }^{12}$, alergia a los metales, traumatismos y fracturas dentales 13-17, alteraciones periodontales ${ }^{18,19}$, acúmulo de biofilm dental y formación de cálculo, malposición dentaria ${ }^{17}$, aspiraciones e ingestiones ${ }^{20}$, sialorrea ${ }^{21,22}$, corrientes galvánicas ${ }^{23}$, alteraciones del habla, masticación y deglución, lesiones hiperplásicas y epitealización del piercing, desgarros traumáticos ${ }^{24}$, transmisión de enferme- dades como: tuberculosis, tétano, sífilis, hepatitis B, C y $\mathrm{D}$ o $\mathrm{VIH}^{25,26}$.

En el presente reporte de caso se describen las principales características del daño ocasionado en la cavidad bucal por la utilización de un piercing lingual.

\section{Reporte del caso}

Un paciente masculino de 29 años, sin enfermedades sistémicas a considerar, se presentó a la consulta refiriendo que se le fracturó la corona de los dientes 3.6 y 4.6 debido a que tenía un piercing en la lengua.

Al examen clínico se observó en la lengua un espacio en la parte dorsal y línea media causada por el piercing; sin embargo, se presentó sin el piercing a la hora de la evaluación (Figura 1).

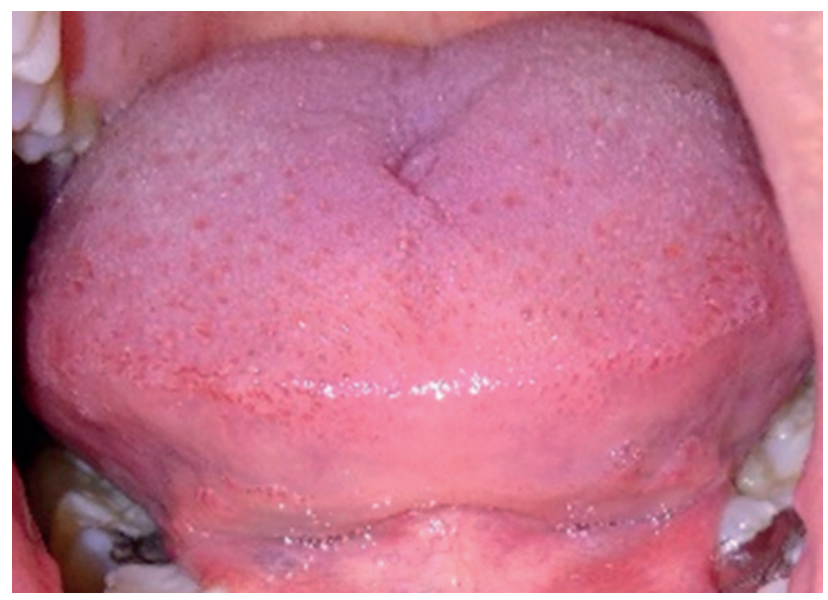

Figura 1. Cicatriz en el dorso de lengua por la utilización de un piercing lingual por más de un año

El diente 4.6 presentó una restauración extensa, sin dolor a la percusión o palpación, movilidad y una bolsa periodontal de $5 \mathrm{~mm}$ por distal, fractura de cúspides vestibulares y caries recidivante (Figura 2A). Radiográficamente se observó un tratamiento de conductos sin lesión periapical y un defecto óseo vertical por distal (Figura 2B).

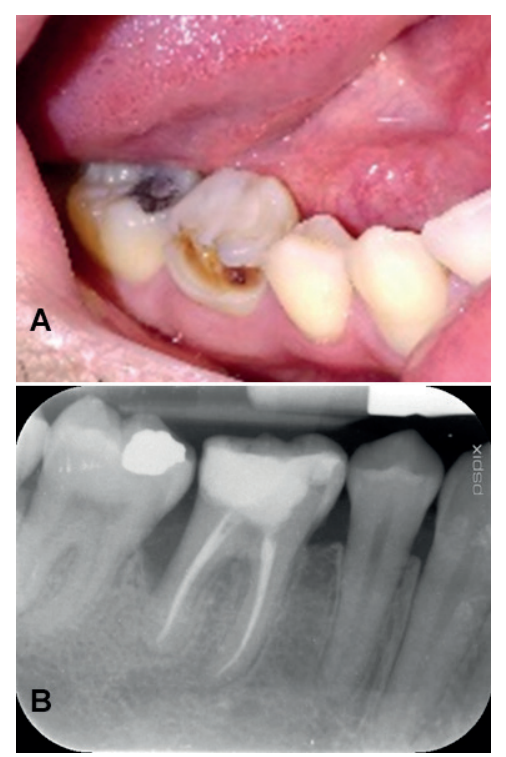

Figura 2. Pieza 4.6 A) Restauración extensa fracturada y caries recidiva por vestibular. B) Se observa un defecto óseo vertical por distal y un tratamiento de conducto en buen estado 
El diente 3.6 presentó una restauración extensa y cúspides vestibulares fracturadas así como caries recidivante (Figura 3A). A las pruebas térmicas presentó sintomatología dolorosa, no hubo molestia a la percusión ni a la palpación, profundidad de sondaje normal, sin movilidad; se diagnosticó pulpitis irreversible en el que indicó tratamiento de conductos. Radiográficamente no se observó ninguna patología periapical (Figura 3B).

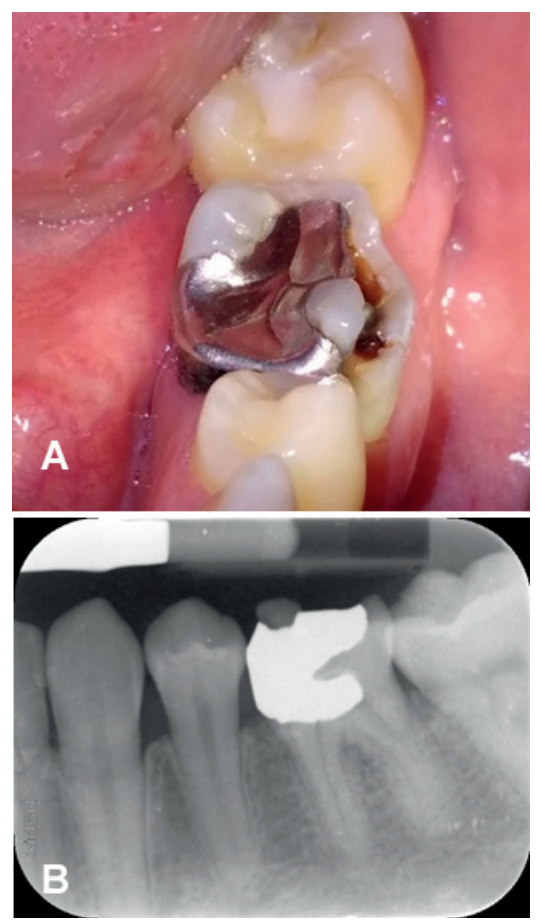

Figura 3. Pieza 3.6 A) Se observa una amalgama extensa fracturada con caries recidivante. B) Radiográficamente no se observa lesión periapical

El diente 4.1 clínicamente presentó una recesión gingival tipo IV de Miller por lingual con bolsa periodontal de $7 \mathrm{~mm}$, movilidad grado I, encía eritematosa y dolor al sondaje (Figura 4A y 4B), radiográficamente se observó una pérdida ósea vertical por distal (Figura $4 \mathrm{C}$ ). Los dientes 4.1 y 4.2 no presentaron signos y síntomas durante las pruebas de sensibilidad térmica.
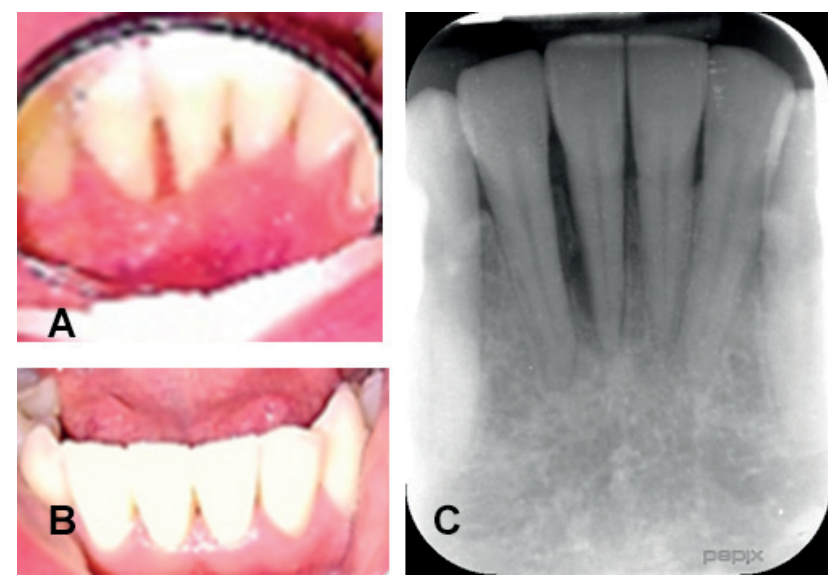

Figura 4. Vista clínica de la región antero inferior. A) Vista lingual de incisivos inferiores, se puede observar una recesión gingival. B) Vista vestibular de piezas anteriores. C) Radiografía del sector anterior, se observa defecto un óseo vertical por distal del diente 4.1
El paciente refirió que el piercing lo utilizó por más de un año, mencionó que era de tipo Barbell. Mencionó que tenía dos hábitos perjudiciales de usar el piercing: morder y mover el piercing sobre los dientes anteriores y posteriores.

El diente 3.6 fue tratado endodónticamente donde se localizaron cuatro conductos, (Figura 5A), se procedió a la instrumentación biomecánica con técnica híbrida, dejando un tamaño apical de 35,02 mm en los conductos mesiales y $50,02 \mathrm{~mm}$ en los conductos distales. Se utilizó hipoclorito de sodio ( $\mathrm{NaClO}$ ) 5,25\%, luego

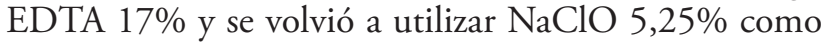
irrigación final, se secó con puntas de papel y se obturó con cemento TOPSEAL ${ }^{\circledR}$ (Dentsply, Maillefer) y técnica lateral. Se colocó ionómero de vidrio y se recomendó realizar una corona definitiva (Figura 5B) y descontinuar el piercing definitivamente.

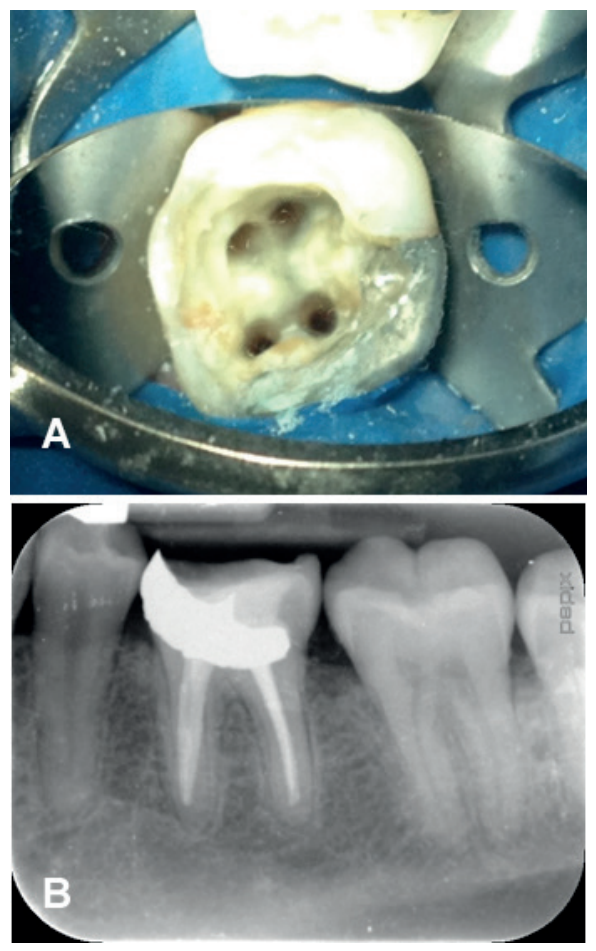

Figura 5. Pieza 3.6 A) Piso de cámara pulpar y entrada de conductos radiculares. B) Radiografía final del tratamiento de conductos

\section{Discusión}

Las perforaciones corporales en los últimos años se han incrementado exponencialmente y se practican cada vez más en personas jóvenes sin distinguir géneros o estratos sociales. El caso clínico presentado representa una situación que puede suceder con mayor frecuencia debido al incremento del uso de los piercings orales en la población.

Se describieron los efectos del piercing lingual sobre los tejidos orales, periodontales e inclusive dentales, evidenciándose recesión gingival, pérdida ósea, daño pulpar y estructural de las piezas dentarias. Un estudio por Campbell et al. ${ }^{27}$, corroboró lo mencionado anteriormente además reveló que el $20 \%$ de sujetos evaluados presentaron recesiones gingivales linguales en incisivos inferiores, Kieser ${ }^{28}$ investigó el trauma periodontal producido 
por el piercing encontrando que en un tercio de ellos al menos tuvo un sitio lingual con recesión gingival. Ziebolz et al. ${ }^{16}$ encontraron una correlación entre el uso del piercing lingual con un incremento en la ocurrencia de fisuras, cracs del esmalte y recesión lingual.

En un estudio hecho en una población universitaria de Finlandia se obtuvieron resultados convincentes en cuanto a la importancia del seguimiento de los piercings en cavidad oral ${ }^{6}$. Se deben evaluar las estructuras circundantes y periorales al piercing como parte de la evaluación periódica en los pacientes ${ }^{29}$, por lo que en el presente caso además del control periodontal y endodóntico, se recomendó que el paciente no vuelva a utilizar el piercing lingual.

El riesgo de infección de un piercing se asocia directamente con la falta de higiene adecuada, técnica de aplicación, lugar donde se coloca, cuidados posteriores, limpieza de la zona y por último con los posibles factores de inmunodepresión ${ }^{24}$. La lengua es un músculo que contiene vasos sanguíneos importantes; cuando se coloca un piercing a nivel lingual se corre el riesgo de exponer estos vasos sanguíneos a abundantes bacterias que viven en la boca. En caso de sufrir una infección, esta puede transmitirse por todo el cuerpo a través de vasos sanguíneos principales. Conociendo el ambiente diverso en microorganismos de la cavidad oral es de suma importancia una buena higiene oral para evitar la formación de biofilm dental, cálculo o caries dental que comprometa las estructuras dentales y periodontales, que junto con malos hábitos con el piercing pueden desencadenar en eventos de mayor importancia clínica.

En la mayor parte de recesiones gingivales linguales y fracturas dentales está involucrado el uso de un piercing lingual, afectando los incisivos centrales inferiores con mayor frecuencia ${ }^{30}$; esto ocurre principalmente por el movimiento de la masticación, el habla o por jugar con el piercing ${ }^{31}$. En el presente caso se observó recesiones gingival linguales en incisivos inferiores, sin embargo, en contradicción con lo anteriormente mencionado se presentó fracturas en primeros molares mandibulares, esto pudo haberse debido a que la estructura dental se vio comprometida por las restauraciones extensas y por la caries recidivantes que presentaron ambos.

Luego de realizar una revisión bibliográfica de las complicaciones de la utilización de los piercings orales, se puede concluir que su presencia y continuo movimiento sobre los tejidos dentales y periodontales puede asociarse con fracturas coronales y problemas periodontales (principalmente recesiones gingivales) como los encontrados en el caso descrito.

\section{Referencias bibliográficas}

1. De Urbiola A, Viñals I. Algunas consideraciones acerca de los piercings orales. Av Odontoestomatol. 2005;21(5):259-69.

2. Ziebolz D, Stuehmer C, van Nuss K, Hornecker E, Mausberg RF. Complications of Tongue Piercing: A Re- view of the Literature and Three Case Reports. J Cont Dent Pract. 2009;10(6):65-71.

3. Jiménez-Nieves HS, Córdoba-Romero IE, Gutiérrez-Rojo JF. Piercings Orales. Rev Tamé. 2015;4(10):365-9.

4. Palacios-Sánchez B, Cerero-Lapiera R, Campo-Trapero J, Esparza-Gómez G. Oral piercing: dental considerations and the legal situation in Spain. Int Dent J. 2005;57(2):60-4.

5. Levin L, Zadik Y, Becker T. Oral and dental complications of intra-oral piercing. Dent Traumatol. 2005;21(6):341-3.

6. Venta I, Lakoma A, Haahtela S, Jaakko P, Ylipaavalniemi P, Turtola L. Oral piercings among first-year university students. Oral Surg Oral Med Oral Pathol Oral Radiol Endod. 2005;99(5):546-9.

7. Chambrone L, Chambrone L. Gingival recessions caused by lip piercing: case report. J Can Dent Assoc. 2003;69(8):505-8.

8. Er N, A-zkavaf A, Berberoglu A, Yamalik N. An unusual cause of gingival recession: oral piercing. J Periodontol. 2000;71(11):1767-9.

9. Peticolas T, Tilliss T, Cross-Poline G. Oral and perioral piercing: a unique form of self-expression. J Cont Dent Pract. 2000;1(3):30-46.

10. Scully C, Chen M. Tongue piercing (oral body art). Br J Oral Maxillofac Surg. 1994;32(1):37-8.

11. Escudero N, Bascones A. Posibles alteraciones locales y sistémicas de los piercings orales y periorales. Av Odontoestomatol. 2007;23(1):21-33.

12. Handrick W, Nenoff P, Mueller H, Knoefler W. Infektionen durch Piercing und Tattoos- eine Uebersicht. Wiener Medizinische Wochenschrif. 2003;153:194-7.

13. De Moor R, De Witte A, De Bruyne M. Tongue piercing and associated oral and dental complications. Endod Dent Traumatol. 2000;16(5):232-7.

14. Krause H, Bremerich A, Sztraka M. Complications following piercing in the oral and facial region. Mund Kiefer Gesichtschir. 2000;4(1):21-4.

15. Boardman R, Smith RA. Dental implications of oral piercing. J Calif Dent Assoc. 1997;25(3):200-7.

16. Ziebolz D, Hildebrand A, Proff P, Rinke S, Hornecker E, Mausberg R. Long-term effects of tongue piercing - a case control study. Clin Oral Invest. 2012;16(1):231-7.

17. Fehrenbach M. Tongue piercing and potential oral complications. J Dent Hyg. 1998;72(1):23-5.

18. Brooks J, Hooper K, Reynolds M. Formation of mucogingival defects associated with intraoral and perioral piercing. JADA. 2003;134(7):837-43.

19. Kretchmer M, Moriarty J. Metal piercing through the tongue and localized loss of attachment: a case report. J Periodontol. 2001;72(6):831-3.

20. De Moor R, De Witte A, Delmé K, De Bruyne M, Hommez G, Goyvaerts D. Dental and oral complications of lip and tongue piercings. Br Dent J. 2005;199(8):506-9.

21. Maibaum W, Margherita V. Tongue piercing: a concern for the dentist. Gen Dent. 1997;45(5):495-7. 
22. Hardee P, Mallya L, Hutchinson I. Tongue piercing resulting in hypotensive collapse. $\mathrm{Br}$ Dent $\mathrm{J}$. 2000;188(12):657-8.

23. Chung MK, Chung D, LaRiccia PJ. Tongue piercing and chronic abdominal pain with nausea and vomiting-two cases. Explore (NY). 2015;11(1):59-62.

24. Mangas C. Complicaciones más frecuentes de piercings y tatuajes. Form Med Cont Aten Prim. 2006;13(9):560-5.

25. Carroll S, Riffenburgh R, Roberts T, Myhre E. Tatoos and body piercings as indicators of adolescent risk-taking behaviors. Pediatrics. 2002; 109(6):1021-7.

26. Alter M. Prevention of spread of Hepatitis C. Hepatology. 2002;36(5):93S-8S.

27. Campbell A, Moore A, Williams E, Stephens J, Takakis D. Tongue piercing: impact of time and barbell stem length on lingual gingival recession and tooth chipping. J Periodontol. 2002;73(3):289-97.

28. Kieser J, Thomson W, Koopu P, Quick A. Oral piercing and oral trauma in a New Zealand sample. Dent Traumatol. 2005;21(5):254-7.

29. Vozza I, Fusco F, Corridore D, Ottolenghi L. Awareness of complications and maintenance mode of oral piercing in a group of adolescents and young Italian adults with intraoral piercing. Oral Med and Pathol. 2015;20(4):e413-8.

30. Simoes A, Conceicao M, Faria R, Morado M. Prevalência de complicacôes associadas à colocacão de piercings orais. Rev Portug Estomatol, Med Dent Cirug Maxilof. 2014;55(4):243-9.

31. Alegría G, Samara G, López M. Riesgos de la salud por el uso del piercing oral. Cient Dent. 2008;5(2):149-54. 
